\title{
Haftpflichtrecht: Beurteilung der Arbeitsunfähigkeit im Haushalt
}

\section{Iris Herzog-Zwitter}

Dr. iur., Juristin Rechtsdienst der FMH und Bildungsbeauftragte Deutschschweiz Swiss Insurance Medicine (SIM),

wissenschaftliche Mitarbeiterin asim Versicherungsmedizin

Die vorliegende Publikation widmet sich dem Zusammenwirken von Medizin und Recht bei der Beurteilung des Haushaltschadens im Haftpflichtrecht. Das Thema Haushaltschaden akzentuiert sich darin, dass unbestritten ein Spannungsfeld zwischen Medizin und Recht gegeben ist. Nicht nur die Beurteilung der Arbeitsunfähigkeit im Erwerb, sondern auch die Beurteilung der Arbeitsunfähigkeit im Haushalt stellt im Einzelfall sowohl an den Mediziner als auch an den Juristen Herausforderungen dar. Die Autoren der Publikation Beurteilung der Arbeitsunfähigkeit - anspruchsvoller Routinevorgang kommen zum Schluss, dass "AUF-Beurteilungen* immer wieder für Verwirrung» sorgen [1]. Diese Aussage gilt nicht nur für AUF-Beurteilungen im Erwerb, sondern auch für den Haushalt.

\section{Einleitung}

Der Haushaltschaden ist ein haftpflichtrechtlicher Fachbegriff und steht oftmals bei den haftpflichtrechtlichen Diskussionen als Schadensposition zur Diskussion, sofern beim Geschädigten bzw. bei der Geschädigten nach dem Haftpflichtereignis eine gesundheitliche Einschränkung im Haushalt vorhanden ist. Eine gänzliche oder teilweise AUF, welche kausal auf ein haftpflichtrechtliches Ereignis zurückzuführen ist, gibt somit nicht nur Anspruch auf Ersatz der Kosten für den Erwerbsausfall und der Erschwerung des wirtschaftlichen Fortkommens sowie eine Genugtuung, sondern auch für den Ersatz bei einem Haushaltschaden.

Wenn die Haftung in einem konkreten Fall unbestritten ist, bedarf es zeitnah zum Haftpflichtereignis einer medizinischen Beurteilung der gesundheitlichen Beeinträchtigung im Haushalt, um den konkreten Schaden erfassen zu können. Ebenso benötigt der Jurist medizinische Beurteilungen der AUF im Haushalt, um den Haftpflichtfall lege artis abwickeln zu können. In solchen Fällen kann man als Jurist nicht auf zusätzliche medizinische Beurteilungen wie zum Beispiel der Invalidenversicherung (IV) zurückgreifen, da diese Abklärungen zu einem viel späteren Zeitpunkt erfolgen. Wenn erst nach Jahren die Haftung nach einem Haft- pflichtereignis feststeht, kann durchaus die Beurteilung der gesundheitlichen Beeinträchtigung in der Haushaltführung durch die IV als zusätzliche medizinische Grundlage dienen.

\section{Aufgabe des Gutachters}

Nach der höchstrichterlichen Rechtsprechung ist die gesundheitliche Einschränkung in der Haushaltführung durch den Mediziner festzulegen. Wie dargelegt benötigt der Jurist in vielen Fällen zeitnah nach dem Haftpflichtereignis eine medizinische Beurteilung der gesundheitlichen Beeinträchtigung im Haushalt. Der Jurist führt mit Bezug auf die medizinische Beurteilung die Berechnung des Haushaltschadens durch.

Das Bundesgericht hat mittels seiner Rechtsprechung die Aufgaben des Gutachters definiert. Diese Präzisierungen gelten auch bei der Beurteilung der AUF im Haushalt. Das Bundesgericht präzisiert wie folgt: «Sache des (begutachtenden) Mediziners ist es, den Gesundheitszustand zu beurteilen und wenn nötig seine Entwicklung im Laufe der Zeit zu beschreiben, d.h. mit den Mitteln fachgerechter ärztlicher Untersuchung unter Berücksichtigung der subjektiven Beschwerden die Befunde zu erheben und gestützt darauf die Diagnose zu stellen. Hiermit erfüllt der Sachverständige 
seine genuine Aufgabe, wofür Verwaltung und im Streitfall Gericht nicht kompetent sind.» Des Weiteren «nimmt die Arztperson zur Arbeitsunfähigkeit Stellung, d.h. sie gibt eine Schätzung ab, welche sie aus ihrer Sicht so substanziell wie möglich begründet. Schliesslich sind die ärztlichen Angaben eine wichtige Grundlage für die juristische Beurteilung der Frage, welche Arbeitsleistungen der Person noch zugemutet werden können» [2]. Der medizinische Gutachter hat zu beurteilen, welche Arbeitsleistung im Haushalt aufgrund der unfallbedingten gesundheitlichen Beeinträchtigungen dauernd oder vorübergehend nicht mehr ausgeführt werden können. Wenn möglich sollte der Gutachter prozentual angeben, inwieweit gesundheitliche Beeinträchtigungen bei einzelnen Haushalttätigkeiten dauernd vorliegen werden und dadurch nicht mehr durchgeführt werden können. Anhaltspunkte für die Beurteilung des Gutachters, welche Tätigkeitsbereiche im Haushalt zu beurteilen sind, sind als Tabellen der statistischen Datengrundlagen der Schweizerischen Arbeitskräfteerhebung (SAKE) des Bundesamtes für Statistik zu finden [3].

\section{Haushaltschaden}

\section{Entwicklung}

Das Bundesgericht hat mittels Rechtsprechung die Parameter des Haushaltschadens festgelegt. Im Jahr 1982 wurde mit dem Entscheid «Blein» ein Grundsatzurteil gefällt. Bis 2011 unterlag der Haushaltschaden einer dynamischen Rechtsentwicklung [4]. Die Rechtsprechung der letzten Jahre zum Thema Haushaltschaden ist von Stabilität geprägt [5].

\section{Haushaltschaden - ein normativer Schaden}

Das Besondere beim haftpflichtrechtlichen Haushaltschaden ist, dass das Bundesgericht mittels Rechtsprechung den Grundstein für den normativen Schadenbegriff gelegt hat. Damit liegt eine Ausnahme zur Differenztheorie vor und bringt spezielle Parameter mit sich [6]. Demnach wird «der wirtschaftliche Wertverlust, der durch die Beeinträchtigung der Arbeitsfähigkeit im Haushalt entstanden ist, und zwar unabhängig davon, ob dieser Wertverlust zur Anstellung einer Ersatzkraft, zu vermehrtem Aufwand der Teilinvaliden, zu zusätzlicher Beanspruchung der Angehörigen oder zur Hinnahme von Qualitätsverlusten führt, ausgeglichen» [7]. Gemäss bundesgerichtlicher Rechtsprechung ist der Aufwand zu messen, den eine entgeltlich eingesetzte Ersatzkraft verursachen würde [8].

\section{Grundparameter des normativen Haushaltschadens}

Der Berechnung des Haushaltschadens im Haftpflichtrecht sind drei Grundparameter zugrunde zu legen.

\section{Die gesundheitliche Beeinträchtigung, welche auf das haftpflichtrechtliche Ereignis kausal zurückzuführen ist}

Diese Beurteilung hat der medizinische Gutachter durchzuführen. Das Bundesgericht präzisiert die Aufgabe des Gutachters, der die AUF im Haushalt festzustellen hat. Des Weiteren hat der Gutachter die funktionellen Folgen der Gesundheitsschädigung qualitativ zu erfassen und quantitativ einzuschätzen [9].

\section{Validenleistung}

Das Bundesgericht setzte mittels der Rechtsprechung die Parameter bei der Bemessung der für den Haushalt erforderlichen Zeit. «Den für die Erledigung des Haushalts erforderlichen Aufwand kann das Sachgericht entweder ausschliesslich gestützt auf statistische Daten festlegen oder konkret ermitteln (BGE 129 III 135 E. 4.2.1 S. 152)» [10].

In einem weiteren Urteil definiert das Bundesgericht die Bemessung der Validenleistung wie folgt: «Der Haushaltschaden ist soweit möglich konkret zu bemessen. Es ist darauf abzustellen, inwieweit die medizinisch festgestellte Invalidität sich auf die Haushaltführung auswirkt. Zur Substanziierung des Haushaltschadens sind daher konkrete Vorbringen zum Haushalt erforderlich, in dem der Geschädigte lebt, zu den darin übernommenen Aufgaben sowie darüber, inwiefern der Ansprecher durch den Unfall bei diesen Leistungen für den Haushalt tatsächlich beeinträchtigt ist» [11]. Als statistische Basis gelten die SAKE-Tabellen. Diese bilden eine statistische Datengrundlage betreffend Zeitaufwand in den einzelnen Haushaltkategorien. Statistische Eckwerte dienen als Orientierungshilfen für die Beurteilung des Einzelfalles [12]. Die in der SAKE abgebildeten Daten zum Zeitaufwand sagen nichts zur Qualität oder Effizienz der Arbeit aus [13].

\section{Stundenansatz}

Der Richtwert des Stundenansatzes für die Abgeltung des Haushaltschadens basiert auf der Rechtsprechung. Bei der Berechnung des Haushaltschadens, den nach einem Unfall der Haftpflichtige für eine bleibende Einschränkung bei der Haushaltarbeit vergüten muss, ist grundsätzlich eine Reallohnerhöhung von einem Prozent jährlich bis zum Alter $64 \mathrm{zu}$ berücksichtigen [14]. 


\section{Bedeutung der Evaluation der Funktionellen Leistungsfähigkeit (EFL) bei der Beurteilung der Arbeitsfähigkeit und Zumutbarkeit bei der Haushaltarbeit}

Das Bundesgericht erachtet es in manchen Fällen als wünschenswert oder sogar als erforderlich für eine valide Beurteilung der Arbeitsfähigkeit und Zumutbarkeit, neben den medizinischen Befunden und Diagnosen auch eine arbeitsorientierte Evaluation der funktionellen Leistungsfähigkeit durchzuführen. Demnach kann «in einem solchen ergonomischen Assessment anhand von Arbeitssimulationstests (wie Heben und Tragen, Arbeit über Kopfhöhe oder Leitersteigen) das arbeitsbezogene Leistungsvermögen generell und mit Blick auf die angestammte berufliche Tätigkeit konkret beurteilt werden» [15]. Zu berücksichtigen sei «neben der Momentaufnahme auch die zukünftige Entwicklungsperspektive - sei dies hinsichtlich der medizinisch-prognostischen Faktoren oder in Bezug auf die Abschätzung des Rehabilitationspotentials für arbeitsrelevante Verbesserungen" [16].

Das Bundesgericht bestätigte in einem nachfolgenden Urteil, dass die EFL gerade bei Erkrankungen am Bewegungsapparat und bei vorliegend von den Experten ausdrücklich bestätigter Leistungsbereitschaft und fehlender Inkonsistenzen zuverlässige Resultate zum Leistungsvermögen bilde [17]. Das Haushaltassessment ist eine EFL mit haushaltspezifischen Testitems. Insbesondere im Rahmen von polydisziplinären Gutachten kann das Haushaltassessment das arbeitsbezogene Leistungsvermögen generell und mit Blick auf die zukünftige Entwicklung ergänzende medizinische Daten liefern.

Korrespondenz: Iris Herzog-Zwitter FMH / Rechtsdienst Elfenstrasse 18 Postfach 300 $\mathrm{CH}-3000$ Bern lex[at]fmh.ch

\section{Zusammenfassung}

Die ärztliche Beurteilung der AUF im Haushalt der geschädigten Person bildet die Grundlage für den Rechtsanwender, den Haushaltschaden in der Folge berech- nen zu können. Der Mediziner muss sich in seiner Rolle als Gutachter bewusst sein, dass die Anforderungen betreffend den Beweiswert eines Arztberichtes seitens des Bundesgerichts wie folgt definiert wurden: «Hinsichtlich des Beweiswertes eines Arztberichtes ist entscheidend, ob der Bericht für die streitigen Belange umfassend ist, auf allseitigen Untersuchungen beruht, auch die geklagten Beschwerden berücksichtigt, in Kenntnis der Vorakten (Anamnese) abgegeben worden ist, in der Beurteilung der medizinischen Zusammenhänge und in der Beurteilung der medizinischen Situation einleuchtet und ob die Schlussfolgerungen des Experten begründet sind» [18].

\section{Literatur}

1 Baumgartner M, Rudolph R. Beurteilung der Arbeitsunfähigkeit anspruchsvoller Routinevorgang. Schweiz Ärzteztg. 2018;99 (51-52):1847-50.

2 BGE $140 \mathrm{~V} 193$.

3 https://www.bfs.admin.ch/bfs/de/home/statistiken/arbeiterwerb/erhebungen/sake.html (letzter Zugriff am 1.4.2019).

4 Blein/Mercadier BGE 108 II 434 = Pra 1983 Nr. 54.

5 Blein/Mercadier BGE 108 II 434 = Pra 1983 Nr. 54; Basler/Jost (nicht amtl. publ. BGE vom 13.12.1994, publiziert in Pra 1995 Nr. 172); BGE 129 III 135 E. 4.2.1; BGE 131 III 360 E. 8.1.

6 BGE 129 III 331 E. 2.1: Definition der Differenztheorie: «Schaden ist nach ständiger Rechtsprechung des Bundesgerichts die ungewollte Verminderung des Reinvermögens. Er kann in einer Verminderung der Aktiven, einer Vermehrung der Passiven oder in entgangenem Gewinn bestehen und entspricht der Differenz zwischen dem gegenwärtigen Vermögensstand und dem Stand, den das Vermögen ohne das schädigende Ereignis hätte.»

7 Blein/Mercadier BGE 108 II 434 = Pra 1983 Nr. 54; Basler/Jost (nicht amtl. publ. BGE vom 13.12.1994, publiziert in Pra 1995 Nr. 172). 8 BGE 131 III 360 E. 8.1 .

9 BGE 141 V 281.

10 BGE 129 III 135 E. 4.2 .1

11 BGE 4A_37/2011 vom 27.4.2011

12 https://www.bfs.admin.ch/bfs/de/home/statistiken/arbeit-erwerb/erhebungen/sake.html (letzter Zugriff vom 1.4.2019).

13 Referat Jacqueline Schön-Bühlmann. Statistische Datengrundlagen zur Berechnung des Haushaltschadens - Werkstattbericht, 5. Personenschadenforum HAVE 19.1.2006.

14 BGE 132 III 321 E.3.

15 BGE 9C_168/2018 vom 8.5.2018.

16 BGE 8C_547/2018 vom 16.1.2009.

17 BGE 9C_384/2015 vom 21.12.2015.

18 BGE 125 V 351. 